\title{
Optical, Ultraviolet, and Infrared Observations of SN 1993J
}

Alexei V. Filippenko ${ }^{1}$ and Thomas Matheson ${ }^{2}$

1 Department of Astronomy, University of California, 601 Campbell Hall, Berkeley, CA 94720-3411, USA;

alex@astron . berkeley .edu

2 Harvard-Smithsonian Center for Astrophysics, 60 Garden Street, Cambridge, MA 02138, USA

tmatheson@cfa.harvard.edu

Summary. We review the existing set of optical/UV/IR observations of Supernova 1993J, concentrating heavily on optical data because these are by far the most plentiful. Some results from theoretical modeling of the observations are also discussed. SN 1993J has provided the best observational evidence for the transformation of a SN from one spectral type to another, thereby providing a link between Type II and Type Ib supernovae (SNe). This has strengthened the argument that SNe Ib (and, by extension, SNe Ic) are core-collapse events. SN 1993J has remained relatively bright for 10 years; its late-time emission comes from the collision of supernova ejecta with circumstellar gas that was released by the progenitor prior to the explosion. The circumstellar material shows strong evidence of CNO processing.

\section{Introduction}

Supernova (SN) 1993J was visually discovered in the nearby galaxy M81 (NGC 3031; $d=3.6 \mathrm{Mpc}$; 25$]$ ) by Francisco García on 1993 March 28.906 UT [54]. Ten years later, we still vividly remember our excitement after receiving by fax a rough finding chart from the discoverers, via the AAVSO. Ever since SN 1987A, those of us in the northern hemisphere had been eagerly waiting for a supernova in M31, or (more likely) in a somewhat more distant galaxy. What a golden opportunity! Unfortunately, our own robotic SN search, at that time being conducted with a 0.76-m telescope at Leuschner Observatory, missed discovery of SN 1993J because its field of view was somewhat too small to include the position of the supernova when centered on the galaxy nucleus, and because of bad weather [18]. It was a pleasure for both of us to finally meet Mr. García, at this conference.

SN 1993J reached $V=10.8 \mathrm{mag}$ (e.g., [52]), becoming the brightest SN in the northern hemisphere since SN $1954 \mathrm{~A}\left(m_{\mathrm{pg}}=9.95 ;[2,71]\right)$. In terms of observational coverage, both in temporal consistency (almost nightly observations at early times) and in the details of individual observations (including observations with signal-to-noise ratios, spectral resolutions, and wavelength regions not typically found in studies of supernovae), SN 1993J is surpassed 
only by SN 1987A. Early spectra showed an almost featureless blue continuum, possibly with broad, but weak, $\mathrm{H} \alpha$ and He I $\lambda 5876$ lines. This led to a Type II classification $[21,26]$ (see [23] for a general discussion of SN types). Wheeler \& Filippenko [70] present a thorough review of the early work on SN 1993J.

Both the spectra and the light curves of SN 1993J quickly began to indicate that this was not a typical Type II SN. Indeed, the initially unusual light curves and the appearance of He I lines in the spectra were interpreted as evidence that SN 1993J was similar to a SN Ib, with a low-mass outer layer of hydrogen that gave the early impression of a SN II (see discussion and references below). Following [72], it was described as a "Type IIb" SN. This transformation from SN II to nearly SN Ib indicates a common mechanism (core collapse) for these two observationally defined subclasses. SN 1993J is thus one of the most significant $\mathrm{SNe}$ ever studied, not only for its role in linking Types II and Ib (and possibly Ic), but also because it was observed with such great detail.

Here we review observations of SN 1993J at optical, ultraviolet (UV), and infrared (IR) wavelengths, concentrating on optical because of the vast amount of data obtained in this wavelength range. Because of time and space constraints, we exclude light echoes and interstellar absorption lines, which have been used to probe the interstellar medium near SN 1993J. A recent optical spectrum of SN 1993J, obtained with the Keck-I $10 \mathrm{~m}$ telescope 9 years and 11 months after the explosion, is shown in Fig. 1. This is very close to 10 years, so we shall dub this the official "Valencia spectrum of SN 1993J."

\section{Photometry of SN 1993J}

The evolution of the light curve of SN 1993J did not follow either of the two typical paths for SNe II. SN 1993J did not remain at a relatively constant brightness after a slight decline from maximum, as a normal Type II plateau SN would, nor did the brightness decline in the pattern of a Type II linear SN. (For representatives of these Type II light curves, see, for example, [13].) Instead, SN 1993J rose quickly, then rapidly declined for $\sim 1$ week, only to brighten a second time over the next two weeks. This led to another rapid decrease in brightness for $\sim 3$ weeks, followed by an approximately exponential decline. For a complete discussion of the photometry of SN 1993J, see [3, 7 , $14,35,46,49,52,53,56,64,69]$.

The unusual initial behavior of the light curve rapidly led many SN modelers to conclude that SN 1993J was the result of a core-collapse explosion in a progenitor that had lost a significant fraction of its hydrogen envelope, leaving only $\sim 0.1-0.5 M_{\odot}$ of hydrogen. The original envelope could have been lost through winds [29] from a fairly massive star $\left(25-30 M_{\odot}\right)$. Another possibility explored by [28] (see also, [43]) is that SN 1993J was the result of 


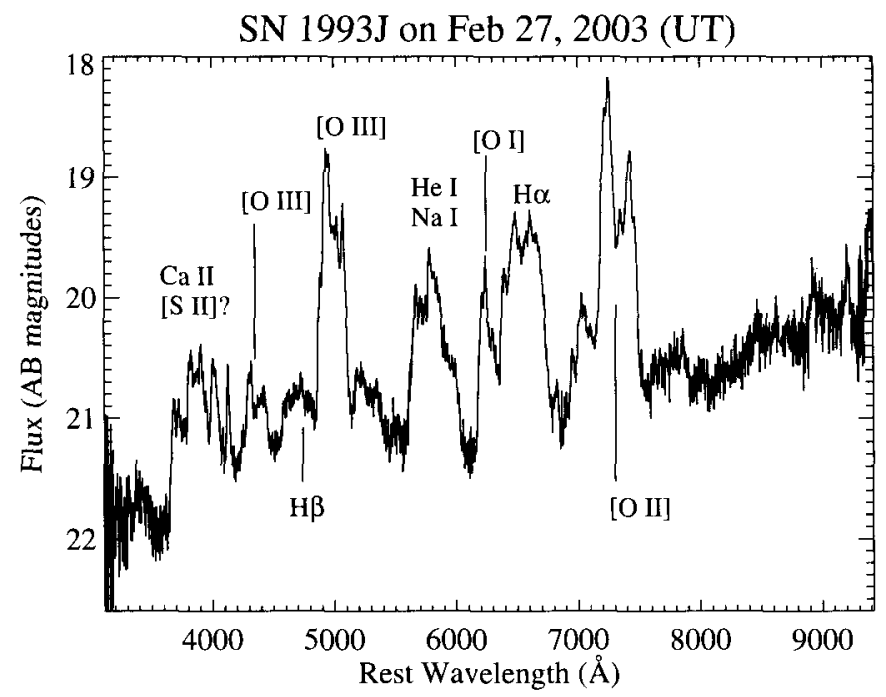

Fig. 1. Keck-I LRIS [45] spectrum of SN 1993J, obtained by A. V. Filippenko and $\mathrm{R}$. Chornock nearly 10 years after the outburst. The absolute flux scale is only approximate, but relative fluxes should be accurate. Plausible line identifications are labeled. $\mathrm{AB}$ mag $=-2.5 \log f_{\nu}-48.6$, on the ordinate scale. (Also known as the "Valencia spectrum of SN 1993.J.")

the explosion of an asymptotic giant branch star having main-sequence mass $M_{m s} \approx 7-10 M_{\odot}$, with a helium-rich envelope.

A more likely solution is that the progenitor of SN 1993J was a member of a binary system and the companion had stripped away a considerable amount of hydrogen. The progenitor was observed during prior studies of M81. Aldering, Humphreys, \& Richmond [1] analyzed several sets of preexisting images and deduced that the photometry was inconsistent with a single star at the position of SN 1993J. They found that the best fit for the progenitor itself was a $\mathrm{K} 0 \mathrm{I}$ star with $M_{\mathrm{bol}} \approx-7.8 \mathrm{mag}$ and $V-R \approx 0.7$ mag. Cohen, Darling, \& Porter [12] derived a similar color from a five-month series of images of M81 from 1984; there was no apparent variability.

Using the scenario of a star that had been stripped of most of its hydrogen envelope, Nomoto et al. [43] and Shigeyama et al. [55] found a best fit to the light curve from their model of a $4 M_{\odot}$ helium core, although a range of 3 $6 M_{\odot}$ for the core is reasonable. The main-sequence mass of the star would have been $15 M_{\odot}$, while the residual hydrogen envelope is less than $\sim 0.9 M_{\odot}$. Starting with a star of initial mass of 13-16 $M_{\odot}$, Woosley et al. [73] could reproduce the light curve from the explosion of a remaining helium core with mass $4.0 \pm 0.5 M_{\odot}$ and hydrogen envelope with mass $0.20 \pm 0.05 M_{\odot}$. A similar model by Podsiadlowski et al. [48] had $0.2 M_{\odot}$ of hydrogen remaining on a 
star of initial mass $M_{i} \approx 15 M_{\odot}$. Ray, Singh, \& Sutaria [51] also invoked a binary system for SN 1993J with a residual hydrogen envelope mass of $0.2 M_{\odot}$. Utrobin [62] used an envelope mass of $0.1 M_{\odot}$ remaining on a $3 M_{\odot}$ helium core from an initial mass of $12 M_{\odot}$. Bartunov et al. [6] achieved a good fit to the light curve with a helium core mass of $3.5 M_{\odot}$, but a larger hydrogen envelope $\left(M_{e n v} \approx 0.9 M_{\odot}\right)$. Later studies continued to conclude that a low-mass envelope of hydrogen on a helium core was the most likely scenario for the progenitor $[63,74]$. Intercomparison of two methods also indicated that the results were robust [8]. Houck \& Fransson [32] used a non-local thermodynamic equilibrium (NLTE) synthetic spectrum code to fit nebular spectra and found that the Nomoto et al. [43] models could explain the late-time spectra. They found a best fit with a $3.2 M_{\odot}$ helium core with a $0.2-0.4 M_{\odot}$ hydrogen envelope. Patat, Chugai, \& Mazzali [47] also used the late-time spectra, specifically the $\mathrm{H} \alpha$ line, to derive an ionized hydrogen mass of $0.05-0.2 M_{\odot}$; this is a lower limit to the envelope mass.

\section{Spectroscopy of SN 1993J}

Woosley et al. [72] had already considered the above possibility for corecollapse SNe, giving them a new name: SNe IIb. The low-mass outer layer of hydrogen would give the initial appearance of a SN II, but the spectrum would slowly change to one more similar to that of a SN Ib, dominated by helium lines with the hydrogen either appearing weakly or completely gone. Indeed, Nomoto et al. [43] predicted that the spectrum of SN 1993J would show this behavior. This was first confirmed by [19], followed rapidly by [56] and [58]. Studies of the early optical spectra include those of $[20,27,42,44,49,59,69]$.

Jeffery et al. [34] present an early UV spectrum of SN 1993J taken with the Hubble Space Telescope (HST) on 1993 April 15 UT. The other corecollapse SNe that had been observed in the UV to that point were compared with SN 1993J, and there were striking differences. SN 1993J had a relatively smooth UV spectrum and was more similar to SN 1979C and SN 1980K, both of which are radio sources and thus likely to have thick circumstellar envelopes (e.g., [68]). The UV spectra of SN 1987A, in contrast, showed broad absorption features. The illumination from circumstellar interaction may reduce the relative strengths of line features compared to the continuum and thus produce the featureless UV spectra of SNe 1979C, 1980K, and 1993J [9].

SN 1993J then evolved fairly rapidly into the nebular phase. The nebularphase spectra were similar to those of a typical SN Ib, but the hydrogen lines never faded completely. In fact, $\mathrm{H} \alpha$ began to dominate the spectrum at late times, certainly the result of circumstellar interaction. There were several other papers that considered the nebular-phase spectra (and some relatively late-time spectra). Filippenko, Matheson, \& Barth [22] show the transition to the nebular phase with spectra from Lick Observatory. Lewis et al. [35] present the complete La Palma archive covering days 2 through 
125. Li et al. [37] discuss the nebular-phase spectra observed from the Beijing Astronomical Observatory. Barbon et al. [3] show the first year of observations from Asiago; the transformation of the SN from Type II to IIb is evident, as is the return of $\mathrm{H} \alpha$ at late times (by $\sim 200$ days). A longer baseline $(\sim 500$ days) for the spectra is found in the work of Finn et al. [24].

There were optical spectropolarimetric observations of SN 1993J. Trammell, Hines, \& Wheeler [60] found a continuum polarization of $P=1.6 \% \pm$ $0.1 \%$ on day 24 (assuming 1993 March 27.5 UT as the explosion date). Trammell et al. [60], as well as later considerations of the same data [30,31], argued that this polarization implied an overall asymmetry, but the source of this asymmetry was undetermined. The presence of SN 1993J in a binary system was implicated as a potential source for the asymmetry. With more epochs of observation, [61] also found a polarization in the continuum of $\sim 1 \%$, but a different level for the interstellar polarization. Nevertheless, they also concluded that SN 1993J was asymmetric. It is interesting to note that a subsequent SN IIb, SN $1996 \mathrm{cb}$, showed substantially similar polarization of its spectra [67].

The analysis of individual aspects of the spectra has yielded some interesting results. Both Wang \& Hu [66] and Spyromilio [57] found evidence for clumpy ejecta with blue shifted emission lines. Houck \& Fransson [32] argue that the lines are not actually blue shifted, but that contamination from other lines appears to shift them. Nonetheless, the lines do show substructure that indicates clumpy ejecta.

Models of the early spectra could reproduce their overall spectral shape, but the line strengths were problematic. Baron et al. [4] found a photospheric temperature of $\sim 8000 \mathrm{~K}$ for day 10 , but the predicted hydrogen and helium lines were too weak, possibly indicating unusual abundances or non-thermal effects. A later analysis including the HST UV spectrum was fit well by including enhanced helium abundance and NLTE effects [5]. Jeffery et al. [34] also had difficulties fitting line strengths for transitions that are susceptible to NLTE effects. Clocchiatti et al. [11] studied the early spectra to follow the evolution of color temperature and to calculate a distance to M81 ( $3.5 \mathrm{Mpc}$ ) using the expanding photosphere method (e.g., [15]. The NLTE treatment of calcium is explored by [75], who found a best fit with a reduced calcium abundance.

\section{Recent Studies of SN 1993J}

Most of the above papers were published before year 2000, but in this section we discuss several more recent studies.

Matheson et al. [39] present a series of 42 Lick and Keck low-resolution optical spectra of SN 1993J from day 3 after explosion to day 2454, as well as one Keck high-dispersion spectrum from day 383. The spectra are studied in detail by [40]. Spectra during the nebular phase, but within the first two 
years after explosion, exhibit small-scale structure in the emission lines of some species, notably oxygen and magnesium, showing that the ejecta of SN $1993 \mathrm{~J}$ are clumpy. On the other hand, a lack of structure in emission lines of calcium implies that the source of calcium emission is uniformly distributed throughout the ejecta. These results are interpreted as evidence that oxygen emission originates in clumpy, newly synthesized material, while calcium emission arises from material pre-existing in the atmosphere of the progenitor [36, 37]. Spectra spanning the range 433-2454 days after the explosion show box-like profiles for the emission lines, clearly indicating circumstellar interaction in a roughly spherical shell. This is interpreted within the Chevalier \& Fransson [10] model for SNe interacting with mass lost during prior stellar winds. At very late times, the emission lines have a two-horned profile, implying the formation of a somewhat flattened or disk-like structure that is a significant source of emission.

Matthews et al. [41] conducted IR photometry (windows in the interval 1.25-3.7 $\mu \mathrm{m}$ ) and IR spectroscopy (windows in the interval $1.2-2.4 \mu \mathrm{m}$ ) of SN $1993 \mathrm{~J}$ at early times, through about day 250 . As in the case of the optical bands, the IR brightness rose to a secondary maximum and then dropped exponentially. However, the $L^{\prime}(3.7 \mu \mathrm{m})$ light curve exhibited an excess, beginning at day 130, which Matthews et al. [41] interpret as thermal emission from dust, as in the SNe II 1987A and 1998S. At early times, during the rise to the secondary maximum, the spectral energy distribution (SED) of SN 1993J could be fitted with black bodies, but such fits were too broad for the observed SEDs during the exponential decline. Though initially featureless, the IR spectra subsequently (during the exponential decline) became dominated by $\mathrm{H}$, He, and probably Fe line emission.

The HST SINS collaboration (Supernova INtensive Study) obtained a series of UV spectra of SN 1993J over the course of about 7 years (Fransson et al. in preparation). It is quite clear that at late times, the emission is almost entirely coming from the shock interaction between the ejecta and circumstellar gas. The model fit to the observed spectrum is quite good, and its total luminosity is fixed by the observed X-ray emission from the shock - it is not a free parameter. Moreover, the detailed line profiles show that the emission is coming from a finite shell rather than a centrally peaked distribution of gas. There are strong, broad emission lines of N II], N III], and N IV], but only weak lines of C III] and C IV - characteristic of CNO processing. Indeed, a model spectrum having $\mathrm{C}: \mathrm{N}=1: 13$ by number in the circumstellar gas (with which the ejecta collide) gives a relatively good fit to the observations. CNO abundance ratios have previously been measured for a number of SNe II, with similar (but perhaps less extreme) results.

From $H S T$ images with 0.05 resolution, Van Dyk et al. [65] identify four stars brighter than $V=25$ mag within $2 . \prime 5$ of SN 1993J that contaminated previous ground-based brightness estimates for the supernova progenitor. Correcting for the contamination, they find that the energy distribution of 
the progenitor is consistent with that of an early K-type supergiant star with $M_{V} \approx-7.0 \pm 0.4 \mathrm{mag}$ and an initial mass of $13-22 M_{\odot}$. The brightnesses of the nearby stars are sufficient to account for the excess blue light seen from the ground in pre-explosion observations [1]. Therefore, the SN 1993J progenitor did not necessarily have a blue companion, although by 2001 , fainter blue stars are seen in close proximity to the supernova. These observations do not strongly limit the mass of a hypothetical companion. A blue dwarf star with a mass up to $30 M_{\odot}$ could have been orbiting the progenitor without being detected in the ground-based images.

Explosion models and observations show that the SN 1993J progenitor had a helium-rich envelope. To test whether the helium abundance could influence the energy distribution of the progenitor, Van Dyk et al. [65] calculated model supergiant atmospheres with a range of plausible helium abundances. The models show that the pre-supernova colors are not strongly affected by the helium abundance longward of $4000 \AA$, and abundances ranging between solar and $\mathbf{9 0 \%}$ helium (by number) are all consistent with the observations.

Recent optical spectra of SN 1993J (e.g., Fig. 1) show that the optical continuum is still very bright $(B \approx V \approx 21 \mathrm{mag})$, certainly from the circumstellar interaction. Van Dyk et al. [65] suggest that the putative, blue companion star has $B>23$ mag and $U>22 \mathrm{mag}$, so perhaps the most likely spectral region in which it might be observed is in the $U$ band, where the actual observed continuum has $U \approx 22 \mathrm{mag}$ (Figure 1 ). At this conference, Stephen Smartt and collaborators reported the possible detection of hydrogen Balmer lines from the putative companion. Given the complexity of the SN spectrum (blends of emission lines, uncertain continuum level), however, this must be verified with future observations; most or all of the claimed Balmer lines may have other explanations. It is also not clear whether the Balmer lines, if real, come from a physical companion star or from an unrelated star along the same line of sight; the HST images shown by Van Dyk et al. [65] reveal several possible contaminants.

\section{Final Remarks}

Although SN 1993J has provided the best observational evidence for the transformation of a SN from one type to another, there have been other examples. The early spectra of SN $1987 \mathrm{~K}$ showed hydrogen lines, but the late-time spectra more closely resembled those of SNe Ib [16]. The transition itself was not observed, occurring while SN $1987 \mathrm{~K}$ was in conjunction with the Sun. SN 1996cb underwent a very similar metamorphosis from SN II to SN Ib; Qiu et al. [50] present a complete spectroscopic record of the transformation. A number of other examples of genuine SNe IIb have been found in recent years. In addition, there were some suggestions of hydrogen in spectra of the Type Ic SN 1987M [17, 33] and the SN Ic 1991A (and perhaps SN Ic 
1990aa [1.7]). SN 1993J is clearly a significant object in the study of SNe. By providing a link between SNe II and SNe Ib, it has strengthened the argument that $\mathrm{SNe} \mathrm{Ib}$ (and, by extension, SNe Ic) are also core-collapse events.

Acknowledgement. A.V.F.'s research on SN 1993J is currently supported by NSF grant AST-0307894, as well as by NASA grants AR-9529 and GO-9114 from the Space Telescope Science Institute, which is operated by AURA, Inc., under NASA contract NAS5-26555. We thank the conference organizers for partial travel funds. We are grateful to the staffs of the Lick and Keck Observatories for help with the observations. The W. M. Keck Observatory is operated as a scientific partnership among Caltech, the University of California, and NASA; the Observatory was made possible by the generous financial support of the W. M. Keck Foundation.

\section{References}

1. G. Aldering, R.M. Humphreys, M. Richmond: Astron. J. 107, 662 (1994)

2. R. Barbon, F. Ciatti, L. Rosino: Astron. Astrophys. 25, 241 (1973)

3. R. Barbon, S. Benetti, E. Cappellaro, F. Patat, M. Turatto, T. Iijima: Astron. Astrophys. Suppl. 110, 513 (1995)

4. E. Baron, P.H. Hauschildt, D. Branch, R.M. Wagner, S.J. Austin, A.V. Filippenko, T. Matheson: Astrophys. J. Lett. 416, L21 (1993)

5. E. Baron, P.H. Hauschildt, D. Branch: Astrophys. J. 426, 334 (1994)

6. O.S. Bartunov, S.I. Blinnikov, N.N. Pavlyuk, D.Yu. Tsvetkov: Astron. Astrophys. 281, L53 (1994)

7. P.J. Benson et al. : Astron. J. 107, 1453 (1994)

8. S.I. Blinnikov, R. Eastman, O.S. Bartunov, V.A. Popolitov, S.E. Woosley: Astrophys. J. 496, 454 (1998)

9. D. Branch, D.J. Jeffery, M. Blaylock, K. Hatano: Pub. Astron. Soc. Pacific 112, $217(2000)$

10. R.A. Chevalier, C. Fransson: Astrophys. J. 420, 268 (1994)

11. A. Clocchiatti, J.C. Wheeler, E.S. Barker, A.V. Filippenko, T. Matheson, J.W. Liebert: Astrophys. J. 446, 167 (1995)

12. J.G. Cohen, J. Darling, A. Porter: Astron. J. 110, 308 (1995)

13. J.B. Doggett, D. Branch: Astron. J. 90, 2303 (1985)

14. V.T. Doroshenko, Yu.S. Efimov, N.M. Shakhovskoi: Astron. Lett. 21, 513 (1995)

15. R.G. Eastman, B.P. Schmidt, R. Kirshner: Astrophys. J. 466, 911 (1996)

16. A.V. Filippenko: Astron. J. 96, 1941 (1988)

17. A.V. Filippenko: Astrophys. J. Lett. 384, L37 (1992)

18. A.V. Filippenko: Sky \& Tel. 86, 30 (1993)

19. A.V. Filippenko, T. Matheson: IAUC 5787 (1993)

20. A.V. Filippenko, T. Matheson, L.C. Ho: Astrophys. J. Lett. 415, L103 (1993)

21. A.V. Filippenko, R.R. Treffers, Y. Paik, M. Davis, E. Schlegel: IAUC 5731 (1993)

22. A.V. Filippenko, T. Matheson, A.J. Barth: Astron. J. 108, 2220 (1994)

23. A.V. Filippenko: Ann. Rev. Astron. Astrophys. 35, 309 (1997) 
24. R.A. Finn, R.A. Fesen, G.W. Darling, J.R. Thorstensen, G.S. Worthey: Astron. J. 110, 300 (1995)

25. W.L. Freedman et al. : Astrophys. J. 427, 628 (1994)

26. P. Garnavich, H.B. Ann: IAUC 5731; corrigendum: IAUC 5733 (1993)

27. P.M. Garnavich, H.B. Ann: Astron. J. 108, 1002 (1994)

28. M. Hashimoto, K. Iwamoto, K. Nomoto: Astrophys. J. Lett. 414, L105 (1993)

29. P. Höflich, N. Langer, M. Duschinger: Astron. Astrophys. 275, L29 (1993)

30. P. Höflich: Astrophys. J. 440, 821 (1995)

31. P. Höflich, J.C. Wheeler, D.C. Hines, S.R. Trammell: Astrophys. J. 459, 307 (1996)

32. J.V. Houck, C. Fransson: Astrophys. J. 456, 811 (1996)

33. D.J. Jeffery, D. Branch, A.V. Filippenko, K. Nomoto: Astrophys. J. Lett. 377, L89 (1991)

34. D.J. Jeffery et al. : Astrophys. J. Lett. 421, L27 (1994)

35. J.R. Lewis et al. : Mon. Not. R. Astron. Soc. 266, L27 (1994)

36. H. Li, R. McCray: Astrophys. J. 387, 309 (1992)

37. A. Li, J. Hu, L. Wang, X. Jiang, H. Li: Astrophys. Space Sci. 211, 323 (1994)

38. H. Li, R. McCray: Astrophys. J. 405, 730 (1993)

39. T. Matheson et al. : Astron. J. 120, 1487 (2000)

40. T. Matheson, A.V. Filippenko, L.C. Ho, A.J. Barth, D.C. Leonard: Astron. J. 120, 1499 (2000)

41. K. Mathews, G. Neugebauer, L. Armus, B.T. Soifer: Astron. J. 123, $753(2002)$

42. N.V. Metlova, D.Yu. Tsvetkov, S.Yu. Shugarov, V.F. Esipov, N.N. Pavlyuk: Astron. Lett. 21, 598 (1995)

43. K. Nomoto, T. Suzuki, T. Shigeyama, S. Kumagai, H. Yamaoka, H. Saio: Nature 364, 507 (1993)

44. K. Ohta, H. Maemura, T. Ishigaki, K. Aoki, H. Ohtani: Pub. Astron. Soc. Japan 46, 117 (1994)

45. J.B. Oke et al. : Pub. Astron. Soc. Pacific 107, 375 (1995).

46. M. Okyudo, T. Kato, T. Ishida, N. Tokimasa, H. Yamaoka: Pub. Astron. Soc. Japan 45, L63 (1993)

47. F. Patat, H. Chugai, P.A. Mazzali: Astron. Astrophys. 299, 715 (1995)

48. Ph. Podsiadlowski, J.J.L. Hsu, P.C. Joss, R.R. Ross: Nature 364, 509 (1993)

49. T.P. Prabhu et al. : Astron. Astrophys. 295, 403 (1995)

50. Y. Qiu, W. Li, Q. Qiao, J. Hu: Astron. J. 117, 736 (1999)

51. A. Ray, K.P. Singh, F.K. Sutaria: Astrophys. Astron. 14, 53 (1993)

52. M.W. Richmond, R.R. Treffers, A.V. Filippenko, Y. Paik, B. Leibundgut, E. Schulman, C.V. Cox: Astron. J. 107, 1022 (1994)

53. M.W. Richmond, R.R. Treffers, A.V. Filippenko, Y. Paik: Astron. J. 112, 732 (1996)

54. J. Ripero, F. García, D. Rodríguez: IAUC 5731 (1993)

55. T. Shigeyama, T. Suzuki, S. Kumagai, K. Nomoto, H. Saio, H. Yamaoka: Astrophys. J. 420, 341 (1994)

56. B.P. Schmidt et al. : Nature 364, 600 (1993)

57. J. Spyromilio: Mon. Not. R. Astron. Soc. 266, L61 (1994)

58. D.A. Swartz, A. Clocchiatti, R. Benjamin, D.F. Lester, J.C. Wheeler: Nature 365, $232(1993)$

59. Y. Taniguchi, T. Murayama, Y. Sato, Y. Yadoumaru, Y. Ohyama, G. Kosugi, M. Yoshida, T. Kurakami: Pub. Astron. Soc. Japan 45, L43 (1993) 
60. S.R. Trammell, D.C. Hines, J.C. Wheeler: Astrophys. J. Lett. 414, L21 (1993)

61. H.D. Tran, A.V. Filippenko, G.D. Schmidt, K.S. Bjorkman, B.T. Jannuzi, P.S. Smith: Pub. Astron. Soc. Pacific 109, 489 (1997)

62. V. Utrobin: Astron. Astrophys. 281, L89 (1994)

63. V.P. Utrobin: Astron. Astrophys. 306, 219 (1996)

64. W. van Driel et al. : Pub. Astron. Soc. Japan 45, L59 (1993)

65. S.D. Van Dyk, P.M. Garnavich, A.V. Filippenko, P. Höflich, R.P. Kirshner, R.L. Kurucz, P. Challis: Pub. Astron. Soc. Pacific 114, 1322 (2002)

66. L. Wang, J. Hu: Nature 369, 380 (1994)

67. L. Wang, D.A. Howell, P. Höflich, J.C. Wheeler: Astrophys. J. 550, 1030 (2000)

68. K.W. Weiler, R.A. Sramek, N. Panagia, J.M. van der Hulst, M. Salvati: Astrophys. J. 301, 790 (1986)

69. J.C. Wheeler et al. : Astrophys. J. Lett. 417, L71 (1993)

70. J.C. Wheeler, A.V. Filippenko: "Review of the Contributions to the Workshop on SN 1993J." In: Supernovae and Supernova Remnants, ed. R.A. McCray, Z. Wang (Cambridge, Cambridge University Press 1996) p. 241

71. P. Wild: Pub. Astron. Soc. Pacific 72, 97 (1960)

72. S.E. Woosley, P.A. Pinto, P.G. Martin, T.A. Weaver: Astrophys. J. 318, $664(1987)$

73. S.E. Woosley, R.G. Eastman, T.A. Weaver, P. Pinto: Astrophys. J. 429, $300(1994)$

74. T.R. Young, E. Baron, D. Branch: Astrophys. J. Lett. 449, L51 (1995)

75. Q. Zhang, Z.R. Wang: Astron. Astrophys. 307, 166 (1996) 